\title{
Adjuvant Treatment after Surgery in Stage IIIA Endometrial Adenocarcinoma
}

\author{
Mee Sun Yoon, MD, PhD ${ }^{1}$ \\ Seung Jae Huh, MD, $\mathrm{PhD}^{2}$ \\ Hak Jae Kim, MD ${ }^{3}$ \\ Young Seok Kim, MD, PhD ${ }^{4}$ \\ Yong Bae Kim, MD, PhD 5 \\ Joo-Young Kim, MD, PhD ${ }^{6}$ \\ Jong-Hoon Lee, $\mathrm{MD}^{7}$ \\ Hun Jung Kim, MD \\ Jihye Cha, MD ${ }^{9}$ \\ Jin Hee Kim, MD, PhD ${ }^{10}$ \\ Juree Kim, MD, PhD'11 \\ Won Sup Yoon, MD, PhD'12 \\ Jin Hwa Choi, MD ${ }^{13}$ \\ Mison Chun, MD, PhD ${ }^{14}$ \\ Youngmin Choi, $\mathrm{MD}^{15}$ \\ Kang Kyoo Lee, MD, PhD ${ }^{16}$ \\ Myungsoo Kim, MD, PhD ${ }^{17}$ \\ Jae-Uk Jeong, MD1 \\ Sei Kyung Chang, MD 18 \\ Won Park, MD, PhD²
}

*A list author's affiliations appears at the end of the paper.

Correspondence: Won Park, MD, PhD

Department of Radiation Oncology,

Samsung Medical Center,

Sungkyunkwan University School of Medicine,

81 Irwon-ro, Gangnam-gu, Seoul 06351, Korea

Tel: 82-2-3410-2616

Fax: 82-2-3410-2619

E-mail: wonro.park@samsung.com

Co-correspondence: Sei Kyung Chang, MD

Department of Radiation Oncology,

CHA Bundang Medical Center, CHA University,

59 Yatap-ro, Bundang-gu, Seongnam 13496,

Korea

Tel: 82-31-780-5426

Fax: 82-31-702-8803

E-mail:skc6603@chamc.co.kr

Received September 18, 2015

Accepted October 7, 2015

Published Online October 29, 2015

\section{Purpose}

We evaluated the role of adjuvant therapy in stage IIIA endometrioid adenocarcinoma patients who underwent surgery followed by radiotherapy (RT) alone or chemoradiotherapy (CTRT) according to risk group.

\section{Materials and Methods}

A multicenter retrospective study was conducted including patients with surgical stage IIIA endometrial cancer treated by radical surgery and adjuvant RT or CTRT. Disease-free survival (DFS) and overall survival (OS) were analyzed.

\section{Results}

Ninety-three patients with stage IIIA disease were identified. Nineteen patients (20.4\%) experienced recurrence, mostly distant metastasis (17.2\%). Combined CTRT did not affect DFS $(74.1 \%$ vs. $82.4 \%, p=0.130)$ or OS $(96.3 \%$ vs. $91.9 \%, p=0.262)$ in stage IIIA disease compared with RT alone. Patients with age $\geq 60$ years, grade G2/3, and lymphovascular space involvement had a significantly worse DFS and those variables were defined as risk factors. The high-risk group showed a significant reduction in 5-year DFS ( $\geq 2$ risk factors) $(49.0 \%$ vs. $88.0 \%, p<0.001)$ compared with the low-risk group $(<2)$. Multivariate analysis confirmed that more than one risk factor was the only predictor of worse DFS (hazard ratio, 5.45; 95\% confidence interval, 2.12 to 13.98; $p$ < 0.001). Of patients with no risk factors, a subset treated with RT alone showed an excellent 5-year DFS and OS (93.8\% and 100\%, respectively).

\section{Conclusion}

We identified a low-risk subset of stage IIIA endometrioid adenocarcinoma patients who might be reasonable candidates for adjuvant RT alone. Further randomized studies are needed to determine which subset might benefit from combined CTRT.
Key words

Endometrial neoplasms, Adjuvant radiotherapy, Adjuvant chemoradiotherapy 


\section{Introduction}

Stage IIIA endometrial cancer represents a wide range of tumor involvement, including the uterine serosa, adnexa, parametria, and peritoneal space, based on the staging system of the International Federation of Gynecology and Obstetrics (FIGO) in 1988 [1-3]. This variability might have an influence on survival for stage IIIA endometrial cancer. In addition, the heterogeneity of histology, such as papillary serous or clear cell carcinoma, makes it difficult to compare outcomes between studies. This variability has led to a wide range of 5-year overall survival (OS) rates for stage IIIA endometrial cancer, varying from $39 \%$ to $76 \%$ [3-6].

The optimal adjuvant management for patients with advanced endometrial cancer has yet to be defined, particularly for IIIA patients as incidence is low, representing only $2.6 \%$ of patients with endometrial cancer [7]. Radiotherapy (RT) has traditionally been employed in advanced endometrial carcinoma to improve locoregional control $[8,9]$. Several studies have evaluated the role of combined modality adjuvant therapy compared with a single modality in advanced endometrial cancer and have suggested that combined chemoradiotherapy (CTRT) can provide additional benefits compared with computed tomography (CT) or RT alone [10-13]. In contrast, a randomized study failed to show an improvement in disease-free survival (DFS) and OS in patients treated with combined CTRT compared with adjuvant RT alone for high-risk endometrial cancer [14]. Those studies included only a small proportion of stage IIIA patients. Thus, the effects of different adjuvant therapies for stage IIIA endometrial cancer remain controversial. In addition, these previous studies involved a small portion of IIIA populations, patient's stage was determined based on the 1988 FIGO stage, and both endometriod and non-endometrioid adenocarcinoma were included.

The current study included a group of patients with stage IIIA endometrioid adenocarcinoma with favorable histology only, using the revised 2009 FIGO stage to overcome the aforementioned variabilities. The revised FIGO IIIA, which was published in 2009, excluded positive peritoneal cytology alone and denoted parametrial involvement separately as stage IIIB. Several studies have attempted to analyze risk factors for poor outcomes $[1,5,6,9,15,16]$; however, few studies have evaluated the role of particular adjuvant therapies according to risk group for revised 2009 FIGO stage IIIA diseases. Here, we evaluated survival outcomes according to risk factors and sought to determine the benefit of combined modality treatment after surgery in the high-risk subset of stage IIIA patients.

\section{Materials and Methods}

All patients with stage IIIA endometrial cancer treated with surgery, followed by adjuvant RT or combined CTRT, in Korea between January 1990 and December 2011 were evaluated. All patients were staged based on the revised 2009 FIGO criteria for endometrial cancer. Patients eligible for analysis had endometrioid adenocarcinoma only.

Nineteen patients with non-endometrioid type adenocarcinoma, such as papillary serous, clear cell, adenosquamous, or carcinosarcoma were excluded. In addition, five patients with only positive peritoneal cytology, and those who underwent adjuvant CT alone $(n=3)$ or inadequate RT $(n=2)$ were also excluded. The remaining 93 stage IIIA patients who underwent surgery followed by adjuvant RT alone or combined CTRT were analyzed retrospectively.

The clinical and pathologic data of 93 patients from 18 institutions were collected. After approval by the Korean Radiation Oncology Group (KROG 13-17), the medical and $\mathrm{RT}$ records of the patients were reviewed retrospectively.

\section{Treatment}

All patients underwent hysterectomy and bilateral salpingo-oophorectomy with pelvic and/ or aortic lymphadenectomy, followed by postoperative RT or combined CTRT. Pelvic lymph node dissection was performed in 77 patients $(82.8 \%)$ and paraaortic lymph node (PALN) dissection or sampling was performed in 31 patients $(33.3 \%)$.

All patients underwent external pelvic RT, delivered to the tumor bed and regional lymphatics with 10-MV photons using the four-field box technique. The total dose to the pelvis ranged from $45 \mathrm{~Gy}$ to $54 \mathrm{~Gy}$ in $1.8 \mathrm{~Gy}$ daily fractions, 5 days per week. Twenty-one patients $(21.6 \%)$ underwent additional vaginal brachytherapy using Fletcher-Suit afterloading applicators. Two to six fractions of 3-5 Gy were delivered to the vaginal surface or $5 \mathrm{~mm}$ from the vaginal surface. Two patients (2.2\%) were treated with an extendedfield RT encompassing a volume of PALN, usually located at the T12-L1 interface. Another two patients received total abdominal RT of 30 Gy in 1.5 Gy fractions followed by a boost to the pelvis with the doses of 19.8 Gy or 24 Gy in 1.8 Gy fractions.

Adjuvant combined CTRT was performed in 36 patients $(38.7 \%)$. Thirty patients were treated with concurrent CTRT (83.3\%), four patients with CT followed by RT $(11.1 \%)$, and two patients with RT followed by CT (5.6\%). Patients receiving adjuvant CT were treated with cisplatin-based CT ( $\mathrm{n}=19$, $52.8 \%)$, carboplatin plus paclitaxel $(n=8,22.2 \%)$, paclitaxel alone $(n=6,16.7 \%)$, or other drugs $(n=3,8.3 \%)$. Adjuvant CT was delivered with a median of six cycles (range, 1 to 9 
cycles), although two patients received only one cycle, and another two patients only received two cycles. Five patients did not complete the full course of CT because of G3 hematologic toxicity $(n=2)$, patient refusal $(n=1)$, or no available data on toxicity $(\mathrm{n}=2)$.

In adjuvant $\mathrm{RT}$ alone, the average treatment period was 42.9 days (range, 36 to 60 days) and 46.9 days (range, 35 to 117 days) for the CTRT group. No significant difference in the RT period was observed between the two treatment groups $(\mathrm{p}=0.057)$.

\section{Statistical analysis}

Locoregional recurrence was defined as a recurrence in the pelvis, vagina, or paraaortic lymphatic region. Failure was defined as biopsy-proven recurrence or progression of disease on serial imaging studies. Time to recurrence and death was calculated from the date of surgery until failure or death from any cause. DFS was defined as alive without disease recurrence at the time of censoring. Survival curves were calculated using the Kaplan-Meier method, and comparison of the curves was performed using a log-rank test. The chisquare test, Fisher exact test, and independent samples $t$ test were used for comparison of characteristics between the two groups. Multivariable analysis was performed using the Cox proportional hazards model. Statistical analyses were performed using SPSS ver. 21 (IBM Co., Armonk, NY), and $\mathrm{p}$-values less than 0.05 were considered statistically significant.

\section{Results}

\section{Patient characteristics and pattern of failure}

The tumor and treatment characteristics of all 93 patients, $57(61.3 \%)$ who received postoperative RT alone and 36 (38.7\%) who received CTRT, are shown in Table 1. The median age of patients in the CTRT group was older than that in the RT alone group. A larger portion of patients in the CTRT group showed involvement of serosa $(27.8 \%$ vs. $19.3 \%$, $\mathrm{p}=0.361)$ and lymphovascular space $(30.6 \%$ vs. $15.8 \%$, $\mathrm{p}=0.214$ ).

A higher proportion of patients in the CTRT group underwent paraaortic lymphadenectomy compared to the RT alone group ( $52.8 \%$ vs. $21.1 \%, \mathrm{p}=0.002)$. The extent of irradiation field of adjuvant RT and vaginal brachytherapy did not differ significantly in the two groups.

The median follow-up period was 62 months (range, 3 to 188 months). Eight patients (14.0\%) in the RT alone group and 11 patients $(30.6 \%)$ in the CTRT group relapsed during the follow-up period $(\mathrm{p}=0.054)$ (Table 2). The majority of recurrences were distant metastases including abdominal recurrence, $75 \%(6 / 8)$ in the RT alone group and $81.8 \%$ $(9 / 11)$ in the CTRT group. In the RT alone group, one patient developed combined local and distant metastases with pelvic lymph node and liver metastasis.

\section{DFS and OS}

The 5-year OS and DFS were $93.7 \%$ and $79.0 \%$, respectively. Univariate analyses showed statistically significant difference in DFS with regard to age $(p<0.001)$, tumor grade $(\mathrm{p}=0.029)$, and lymphovascular involvement $(\mathrm{p}=0.041)$ (Table 3). No significant difference in OS (91.9\% vs. $96.3 \%$, $\mathrm{p}=0.262)$ or DFS $(82.4 \%$ vs. $74.1 \%, \mathrm{p}=0.130)$ was observed between the RT alone group and the combined CTRT group. Five-year DFS was lower in patients with serosal involvement than in those without, but there was no statistical significance $(81.5 \%$ vs. $67.2 \%, \mathrm{p}=0.180)$. Involvement of adnexa did not show a significant relationship with DFS. Of the 93 patients, $57(61.3 \%)$ had solitary adnexal or serosal involvement. Patients with serosal involvement had a worse 7-year DFS (66.7\%) compared to those with solitary adnexal involvement $(90.5 \%)$, but this difference did not reach statistical significance $(\mathrm{p}=0.629)$.

The above mentioned variables (age $\geq 60$ years, tumor grade $2 / 3$, and lymphovascular space involvement) for prognosis in DFS were defined as risk factors. Of 77 patients with available data for risk factors, 25 patients $(32.5 \%)$ had no risk factors, $30(38.9 \%)$ had one, $16(20.8 \%)$ had two, and six $(7.8 \%)$ had three. A significant correlation was observed between the number of risk factors and DFS. The 5-year DFS was $91.7 \%$ in patients with no risk factors, $84.8 \%$ in patients with one, $55.0 \%$ in patients with two, and $33.3 \%$ in patients with three ( $\mathrm{p}=0.001$ ). The 5 -year OS according to the number of risk factors was $95.0 \%$ in patients with no risk factors, 92.0\% in patients with one, $93.8 \%$ in patients with two, and $80.0 \%$ in patients with three $(\mathrm{p}=0.542)$.

The patients were subgrouped according to low-risk (patients with no or one risk factor) and high-risk (patients with two or three risk factors) groups. The proportion of high-risk patients in the combined CTRT group was more than twice that of the RT alone group (41.9\% vs. 19.6\%, $\mathrm{p}=0.033)$. Thirty-seven patients $(80.4 \%)$ in RT alone were classified as low risk and nine (19.6\%) as high risk. In CTRT, 18 patients $(58.1 \%)$ were classified as low risk, while 13 patients $(41.9 \%)$ as high risk. A significant reduction in 5-year DFS was observed in the high-risk group $(49.0 \%$ vs. $88.0 \%$, $\mathrm{p}<0.001)$ compared with the low-risk group (Fig. 1). Five-year OS was not significantly lower in the high-risk group $(90.7 \%)$ versus the low-risk group $(93.5 \%)(p=0.618)$. 
Table 1. Clinical and pathologic characteristics in stage IIIA endometrial adenocarcinoma according to adjuvant modality

\begin{tabular}{|c|c|c|c|c|}
\hline Characteristic & All $(n=93)$ & RT alone $(n=57)$ & CTRT $(n=36)$ & p-value \\
\hline Age, median (range, yr) & $51(25-83)$ & $50(25-83)$ & $54(35-75)$ & 0.281 \\
\hline \multicolumn{5}{|l|}{ Age (yr) } \\
\hline$<60$ & $74(79.6)$ & $46(80.7)$ & $28(77.8)$ & 0.733 \\
\hline$\geq 60$ & $19(20.4)$ & $11(19.3)$ & $8(22.2)$ & \\
\hline \multicolumn{5}{|l|}{ Grade } \\
\hline I & $41(44.1)$ & $28(49.1)$ & $13(36.1)$ & 0.291 \\
\hline II & $30(32.3)$ & $15(26.3)$ & $15(41.7)$ & \\
\hline III & $20(21.5)$ & $12(21.1)$ & $8(22.2)$ & \\
\hline Unknown & $2(2.2)$ & $2(3.5)$ & 0 & \\
\hline \multicolumn{5}{|l|}{ Serosal involvement } \\
\hline Negative & $70(75.3)$ & $44(77.2)$ & $26(72.2)$ & 0.361 \\
\hline Positive & $21(22.6)$ & $11(19.3)$ & $10(27.8)$ & \\
\hline Unknown & $2(2.2)$ & $2(3.5)$ & 0 & \\
\hline \multicolumn{5}{|l|}{ Adnexal involvement } \\
\hline Negative & $16(17.2)$ & $11(19.3)$ & $5(13.9)$ & 0.501 \\
\hline Positive & $77(82.8)$ & $46(80.7)$ & $31(86.1)$ & \\
\hline \multicolumn{5}{|l|}{ Lymphovascular involvement } \\
\hline Negative & $59(63.4)$ & $38(66.7)$ & $21(58.3)$ & 0.214 \\
\hline Positive & $20(21.5)$ & $9(15.8)$ & $11(30.6)$ & \\
\hline Unknown & $14(15.1)$ & $10(17.5)$ & $4(15.1)$ & \\
\hline Tumor size $^{\text {a) }}$, mean (range, cm) & $4.6(0.4-12)$ & $4.4(1.5-12)$ & $5.0(0.4-10.2)$ & 0.327 \\
\hline \multicolumn{5}{|l|}{ Lymph node dissection } \\
\hline Pelvic LN dissection & $77(82.8)$ & $44(77.2)$ & $33(91.7)$ & 0.072 \\
\hline Paraaortic LN dissection & $31(33.3)$ & $12(21.1)$ & $19(52.8)$ & 0.002 \\
\hline \multicolumn{5}{|l|}{ Type of external beam radiotherapy } \\
\hline Whole pelvis & $89(95.7)$ & $54(94.7)$ & $35(97.2)$ & 0.500 \\
\hline Whole pelvis+paraaortic field & $2(2.2)$ & $1(1.8)$ & $1(2.8)$ & \\
\hline Whole abdomen & $2(2.2)$ & $2(3.5)$ & 0 & \\
\hline \multicolumn{5}{|l|}{ Additional vaginal brachytherapy } \\
\hline No & $72(77.4)$ & $46(80.7)$ & $26(72.2)$ & 0.341 \\
\hline Yes & $21(21.6)$ & $11(19.3)$ & $10(27.8)$ & \\
\hline \multicolumn{5}{|l|}{ CTRT sequence } \\
\hline Concurrent & & & $30(83.3)$ & \\
\hline Sequential & & & $6(16.7)$ & \\
\hline
\end{tabular}

Values are presented as number (\%) unless otherwise indicated. RT, radiotherapy; CTRT, chemoradiotherapy; LN, lymph node. ${ }^{\text {a) }}$ Data were available in 70 patients with measurable primary tumor.

For RT alone, 5-year DFS and OS were lower in the highrisk group than in the low-risk group $(66.7 \%$ vs. $84.6 \%$, $\mathrm{p}=0.133$ and $77.8 \%$ vs. $93.5 \%, \mathrm{p}=0.149$, respectively), but the differences did not reach statistical significance (Fig. 2). In the CTRT group, the low-risk group showed an improved 5 -year DFS ( $94.4 \%$ vs. $38.5 \%, \mathrm{p}=0.001)$ compared with the high-risk group, but there was no significant difference in OS (93.3\% vs. $100 \%, \mathrm{p}=0.465)$.

\section{Multivariate analysis}

To examine the role of adjuvant multi-modality therapy, the risk groups were analyzed according to treatment modalities. No significant difference in 5-year OS (93.5\% vs. $93.3 \%$, $\mathrm{p}=0.785)$ or DFS ( $84.6 \%$ vs. $94.4 \%, \mathrm{p}=0.852)$ was observed between the RT alone group and the combined CTRT group in the low-risk group. In the high-risk group, combined CTRT showed a trend for improved 5-year OS (100\% vs. $77.8 \%, \mathrm{p}=0.086$ ) compared with RT alone, but the difference did not reach statistical significance. Combined CTRT did 
Table 2. Patterns of failure according to adjuvant treatment

\begin{tabular}{|c|c|c|c|}
\hline Treatment & RT alone $(\mathrm{n}=57)$ & CTRT $(\mathrm{n}=36)$ & p-value ${ }^{a}$ \\
\hline Locoregional recurrence & - & $2(18.2)$ & - \\
\hline Pelvic cavity only & - & 1 & \\
\hline Pelvic LN+vagina & - & 1 & \\
\hline Pelvic LN+paraaortic LN & $1(12.5)$ & - & \\
\hline Distant metastasis & $6(75.0)$ & $9(81.8)$ & - \\
\hline Peritoneal seeding only & 2 & 2 & \\
\hline \multicolumn{4}{|l|}{ Solid organs (liver, lung, vulva, etc.) } \\
\hline Lung only & 1 & 4 & \\
\hline Lung+other organ & 1 & 1 & \\
\hline Vulva & 1 & 1 & \\
\hline Other or multiple (liver, abd wall, and spleen) & 1 & 1 & \\
\hline Combined LRR and DM & $1(12.5)$ & - & - \\
\hline Total recurrence & $8(14.0)$ & $11(30.6)$ & 0.054 \\
\hline
\end{tabular}

Values are presented as number (\%). RT, radiotherapy; CTRT, chemoradiotherapy; LN, lymph node; LRR, locoregional recurrence; DM, distant metastasis. ${ }^{\text {a) }}$ Chi-square test.

Table 3. Factors affecting survival in univariate analysis

\begin{tabular}{|c|c|c|c|c|}
\hline \multirow{2}{*}{ Parameter } & \multicolumn{2}{|c|}{ Disease-free survival } & \multicolumn{2}{|c|}{ Overall survival } \\
\hline & 5 -Year $(\%)$ & p-value & 5 -Year $(\%)$ & p-value \\
\hline \multicolumn{5}{|c|}{ Age ( $<60$ yr vs. $\geq 60 \mathrm{yr})$} \\
\hline$<60$ & 86.6 & $<0.001$ & 96.7 & 0.028 \\
\hline$\geq 60$ & 48.9 & & 81.4 & \\
\hline \multicolumn{5}{|c|}{ Grade (G1 vs. G2/3) } \\
\hline G1 & 90.0 & 0.029 & 94.4 & 0.387 \\
\hline $\mathrm{G} 2 / \mathrm{G} 3$ & 68.9 & & 93.3 & \\
\hline \multicolumn{5}{|l|}{ LVI } \\
\hline No & 83.2 & 0.041 & 91.9 & 0.699 \\
\hline Yes & 57.9 & & 94.4 & \\
\hline \multicolumn{5}{|c|}{ Serosal involvement } \\
\hline No & 81.5 & 0.180 & 91.8 & 0.747 \\
\hline Yes & 67.2 & & 100 & \\
\hline \multicolumn{5}{|c|}{ Adnexal involvement } \\
\hline No & 73.8 & 0.790 & 100 & 0.991 \\
\hline Yes & 79.1 & & 92.5 & \\
\hline \multicolumn{5}{|c|}{ Size (diameter, $\mathrm{cm}$ ) } \\
\hline$\leq 5$ & 84.0 & 0.175 & 95.5 & 0.482 \\
\hline$>5$ & 77.3 & & 89.4 & \\
\hline \multicolumn{5}{|c|}{ Adjuvant treatment } \\
\hline RT alone & 82.4 & 0.130 & 91.9 & 0.262 \\
\hline CTRT & 74.1 & & 96.3 & \\
\hline
\end{tabular}

LVI, lymphovascular involvement; RT, radiotherapy; CTRT, chemoradiotherapy.

not affect 5-year DFS (38.5\% vs. 66.7\%, p=0.274) compared with RT alone for the high-risk group.

In multivariate analysis including age, grade, lympohovascular involvement, number of risk factors, and type of adju- vant modality, more than one risk factor was the only predictor of worse DFS (hazard ratio [HR], 5.45; 95\% confidence interval [CI], 2.12 to 13.98; $\mathrm{p}<0.001)$. In multivariate analysis for OS, only old age was predictive of OS (HR, 5.8; 95\% CI, 

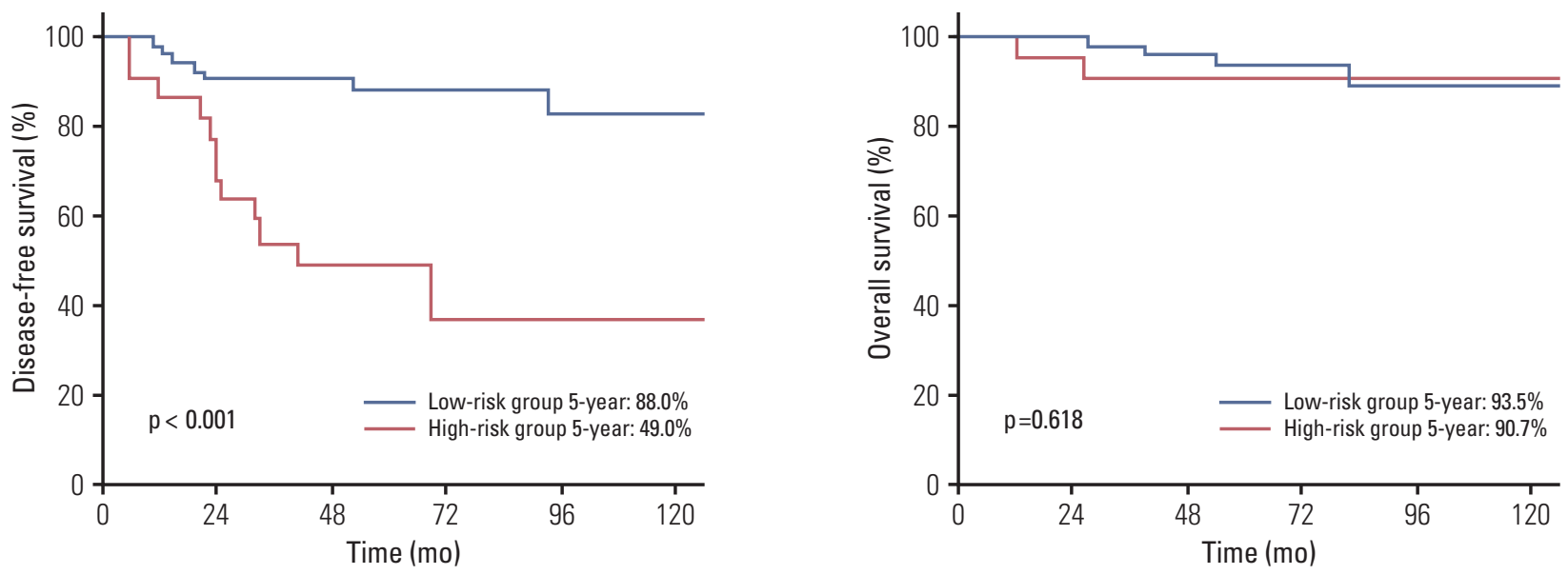

Fig. 1. Kaplan-Meier survival curve of disease-free survival (A) and overall survival (B) according to the low-risk group with no or one risk factor and the high-risk group with two or three risk factors (risk factor: age $\geq 60$ or $\geq$ G2 or lymphovascular space invasion).

1.15 to $29.61 ; \mathrm{p}=0.034)$. In multivariate analysis, combined CTRT did not show a statistical difference compared to RT alone in DFS or OS (Table 4).

\section{Treatment related toxicities}

Twelve patients $(33.3 \%)$ in the CTRT group experienced grade 3-4 acute toxicity. Grade 3-4 acute hematologic toxicities were observed in 11 patients in the CTRT group and a grade 3 acute intestinal obstruction was found in one patient. In the RT alone group, one patient $(1.8 \%)$ treated with extended-field RT had grade 4 acute hematologic toxicity. No patients in the CTRT group had grade 3-4 late toxicity and one patient in the RT alone group treated with whole abdominal irradiation had a grade 3 late leg edema.

\section{Discussion}

The current study showed the impact of a number of risk factors for stage IIIA endometrioid adenocarcinoma. Patients with more than one risk factor (high-risk group) had a worse prognosis. Indeed, more than one risk factor was the only strong negative prognostic factor in multivariate analysis. Patients in the high-risk group were 4 to 5 times more likely to develop a distant metastasis compared to those in the lowrisk group ( $40.9 \%$ vs. $9.1 \%, \mathrm{p}<0.001)$, therefore, the addition of more intense systemic therapy to those in the high-risk group may improve the outcome. The current study showed that combined CTRT showed a trend with an improved 5-year OS (100\% vs. $77.8 \%, \mathrm{p}=0.086)$ compared with RT alone, although CTRT did not affect 5-year DFS compared with RT alone for the high-risk group. Several studies have suggested that combined CTRT can provide additional benefits compared with CT or RT alone [10-13]. The retrospective study in stage III and IV endometrial cancer by Alvarez Secord et al. [13] reported that adjuvant CT alone was associated with poor 3-year OS (33\%) and progression-free survival (19\%) compared to either RT alone (70\% and 59\%) or combined therapy (79\% and 62\%). In a multicenter retrospective study conducted with 78 patients with stage III endometrial cancer treated with adjuvant CT and/or RT, the 3-year relapse-free survival rates were $86.5 \%$ for the combined CTRT group, $65.8 \%$ for the CT alone, and $44.1 \%$ for RT alone, suggesting that combined CTRT modality may induce an advantage in relapse-free survival compared to RT or CT alone [17]. Randomized trials were conducted for comparison of sequential CTRT with RT alone. The first trial was run by the Italian Oncology Group with 153 patients (IIB-IIIC, $65 \%$ stage III), and the second by the Nordic Society of Gynaecological Oncology/European Organisation for the Research and Treatment of Cancer, which included patients with mainly high-risk early stage disease (only $1.6 \%$ stage III). In the Italian trial, no statistically significant difference in DFS or OS was observed between treatment groups. However, in joint pooled analysis in these two randomized trials, 

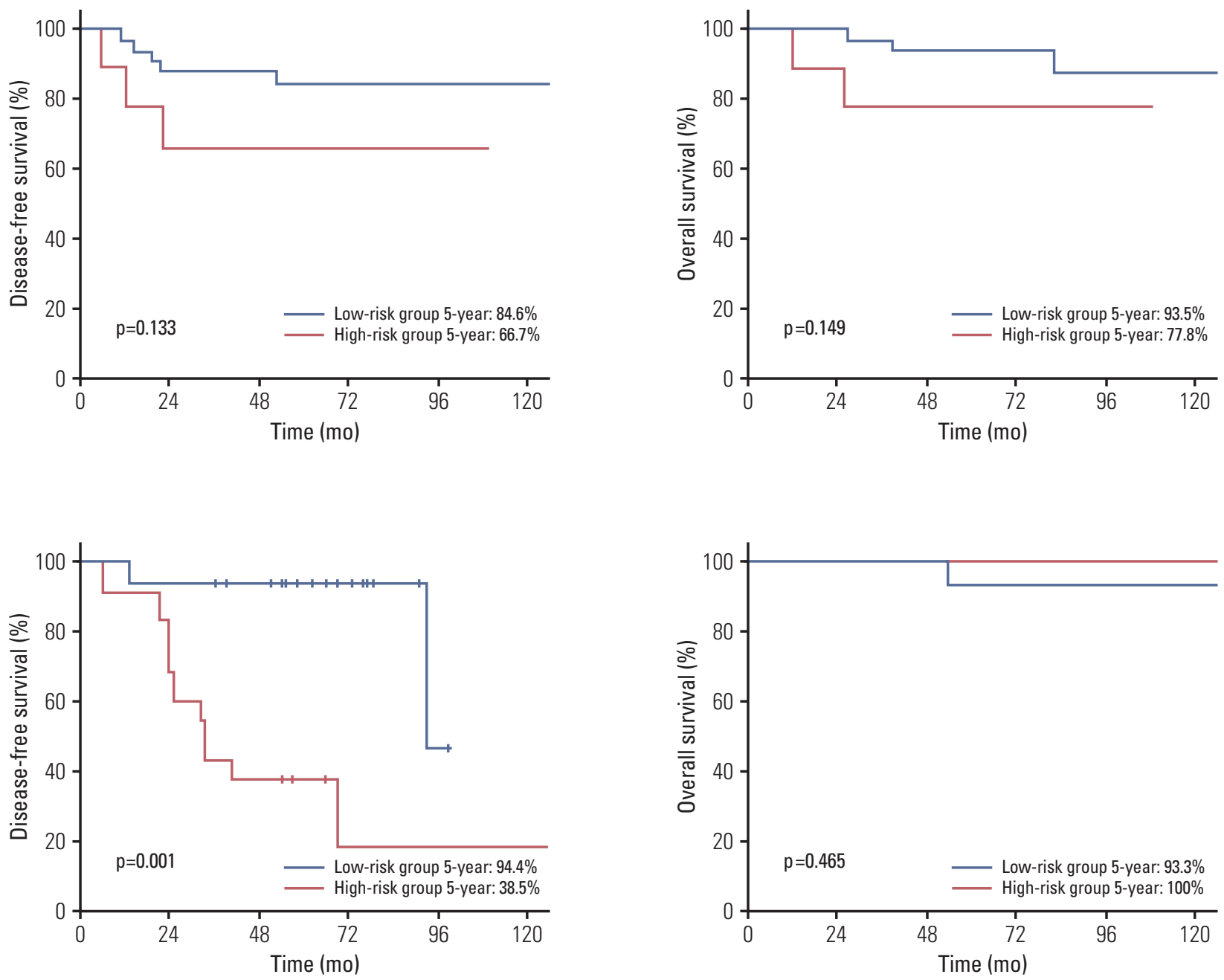

Fig. 2. Subgroup analysis according to treatment modality. Disease-free survival and overall survival in the radiotherapy alone group (A) and the chemoradiotherapy group (B). The low-risk group with no or one risk factor and the high-risk group with two or three risk factors (risk factor: age $\geq 60$ years or $\geq \mathrm{G} 2$ or lymphovascular space invasion).

Table 4. Multivariate analysis

\begin{tabular}{lcccc}
\multirow{2}{*}{ Parameter } & \multicolumn{2}{c}{ Disease-free survival } & \multicolumn{2}{c}{ Overall survival } \\
\cline { 2 - 3 } \cline { 5 - 6 } Age $(<60$ yr vs. $\geq 60$ yr $)$ & HR $(95 \%$ CI $)$ & p-value & HR (95\% CI) & p-value \\
Grade (G1 vs. G2G3) & - & 0.340 & $5.83(1.15-29.61)$ & 0.034 \\
LVI (no vs. yes) & - & 0.903 & - & 0.834 \\
No. of risk factors ${ }^{a}(<2$ vs. $\geq 2)$ & $5.45(2.12-13.98)$ & $<0.001$ & - & 0.437 \\
RT alone vs. CTRT & - & 0.488 & - & 0.177 \\
\hline
\end{tabular}

$\mathrm{HR}$, hazard ratio; CI, confidence interval; LVI, lymphovascular space invasion; RT, radiotherapy; CTRT, chemoradiotherapy.

a)Risk factor: age $\geq 60$ years or $\geq \mathrm{G} 2$ or LVI. 
sequential addition of CT to RT improved at least progression-free survival (78\% vs. $69 \%, \mathrm{p}=0.009)$, and trended a benefit of overall survival ( $82 \%$ vs. $75 \%$, $\mathrm{p}=0.07$ ) compared with RT alone [12].

An important question is whether or not it is reasonable to recommend adjuvant $\mathrm{RT}$ alone without chemotherapy in low-risk patients. Prior study has suggested that a subgroup of women with stage IIIA who have endometrioid tumor, no lymphovascular space involvement, and positive peritoneal cytologic finding only show an excellent prognosis [15]. Havrilesky et al. [6] found that among 24 patients with peritoneal cytology only, non-serous histology, and grade 1-2 disease, no recurrence was found in 12 patients receiving adjuvant treatment, while one patient showed recurrence without adjuvant treatment. Our study included patients according to the 2009 FIGO staging system and excluded those with peritoneal cytology only. For 25 patients with no risk factors, 17 patients $(68 \%)$ were treated with adjuvant RT alone and eight patients (32\%) with adjuvant combined CTRT, resulting in excellent 5-year DFS (91.7\%) and OS $(95.0 \%)$, respectively. No difference in DFS and OS was observed between RT alone and the CTRT group. Thus, a subset of stage IIIA patients with no risk factors might be treated with adjuvant RT alone.

Several studies have reported that patients with extrauterine spread limited to the adnexa showed favorable 5-year DFS rates, ranging from $71 \%$ to $89.6 \%[3,18,19]$. By contrast, serosal involvement had a relatively poor prognosis $[15,20]$. A retrospective study of 15 patients with solitary serosal invasion had a poor 5-year DFS (41.5\%) due to the high incidence of distance metastases [20]. A multicenter retrospective comparative analysis in Netherlands published the outcomes for 67 patients with stage IIIA endometrial carcinoma, evaluating differences in outcome between serosa and adnexal involvement [18]. Those with involvement of the serosa alone had a worse 7-year distant metastasis-free survival $(58.7 \%)$ compared to adnexal involvement alone $(72.7 \%)$, but this difference was not significant $(\mathrm{p}=0.399)$. Authors concluded that either adnexal or serosal involvement showed a comparable disease-specific survival and only presence of lymphovascular invasion was significant in multivariate analysis (HR, 3.6; $\mathrm{p}=0.038$ ). The current study was composed of a relatively large homogeneous subgroup of surgically staged IIIA patients with only adnexal or serosal involvement. Of the 93 patients in our study, 57 (61.3\%) had solitary adnexal or serosal involvement. Consistent with previous studies, patients with serosal involvement had a worse 7-year DFS (66.7\%) compared to those with solitary adnexal involvement $(90.5 \%)(\mathrm{p}=0.629)$.

In this multicenter analysis, we failed to demonstrate additional benefit of combined adjuvant CTRT compared with adjuvant RT alone for stage IIIA endometrial cancer.
While these findings might suggest a comparable survival outcome between combined CTRT and RT alone groups, our study is limited by the difference of high-risk features between the groups. Although there was no statistically significant difference in median age, grade, and lymphovascular involvement between the RT alone group and the combined CTRT group, the CTRT groups did show a trend with an older age, higher grade, and more involvement of lymphovascular space than the RT alone group. In addition, a subset of patients with more than one risk factor, the highrisk group, in the combined CTRT group was more than twice that of the RT alone group (41.9\% vs. $19.6 \%, \mathrm{p}=0.033$ ). These factors might lead to comparable survival outcomes between the RT alone group and the combined CTRT group. Another limitation of this study is the retrospective design with heterogeneity of the $\mathrm{CT}$ agent, sequence of combined CTRT and surgical technique with paraaortic lymphadectomy. This variability leads to difficulty in determining the additional benefit of CTRT in stage IIIA endometrioid adenocarcinoma.

For gynecologic Oncology group 258 (GOG258), the current phase III randomized trial for adjuvant modality, the CTRT followed by carboplatin+paclitaxel is being compared to combination of carboplatin and paclitaxel in stage III and IVA patients. Multi-center randomized trials are required to further evaluate the role of adjuvant combined modality, according to risk groups, particularly in stage IIIA endometrial adenocarcinoma which is an uncommon entity.

\section{Conclusion}

In conclusion, the current study showed a favorable outcome in a subset of low-risk patients compared to the highrisk group in stage IIIA endometrioid adenocarcinoma treated with adjuvant RT alone or adjuvant combined CTRT. We found that patients classified as high risk ( $\geq 2$ risk factors) showed a significant reduction in 5-year DFS compared with the low-risk group $(49.0 \%$ vs. $88.0 \%, \mathrm{p}<0.001)$ with an increase in the development of distant metastasis $(40.9 \%$ vs. $9.1 \%, \mathrm{p}<0.001)$. We identified a subset of stage IIIA patients without risk factors who might be reasonable candidates for adjuvant $\mathrm{RT}$ alone.

\section{Conflicts of Interest}

Conflict of interest relevant to this article was not reported. 


\section{Author Details}

${ }^{1}$ Department of Radiation Oncology, Chonnam National University Hwasun Hospital, Chonnam National University Medical School, Hwasun, ${ }^{2}$ Department of Radiation Oncology, Samsung Medical Center, Sungkyunkwan University School of Medicine, Seoul, ${ }^{3}$ Department of Radiation Oncology, Seoul National University Hospital, Seoul, ${ }^{4}$ Department of Radiation Oncology, Asan Medical Center, Seoul, ${ }^{5}$ Department of Radiation Oncology, Yonsei University College of Medicine, Seoul, ${ }^{6}$ Center for Uterine Cancer, Research Institute and Hospital, National Cancer Center, Goyang, ${ }^{7}$ Department of Radiation Oncology, St. Vincent's Hospital, College of Medicine, The Catholic University of Korea, Suwon, ${ }^{8}$ Department of Radiation Oncology, Inha University School of Medicine, Incheon, ${ }^{9}$ Department of Radiation Oncology,
Yonsei University Wonju College of Medicine, Wonju, ${ }^{10}$ Department of Radiation Oncology, Dongsan Medical Center, Keimyung University School of Medicine, Daegu, ${ }^{11}$ Department of Radiation Oncology, Cheil General Hospital and Women's Healthcare Center, Kwandong University College of Medicine, Seoul, ${ }^{12}$ Department of Radiation Oncology, Korea University College of Medicine, Seoul, ${ }^{13}$ Department of Radiation Oncology, Chung-Ang University Hospital, Seoul, ${ }^{14}$ Department of Radiation Oncology, Ajou University School of Medicine, Suwon, ${ }^{15}$ Department of Radiation Oncology, Dong-A University School of Medicine, Busan, ${ }^{16}$ Department of Radiation Oncology, University of Wonkwang School of Medicine, Iksan, ${ }^{17}$ Department of Radiation Oncology, Incheon St. Mary's Hospital, College of Medicine, The Catholic University of Korea, Incheon, ${ }^{18}$ Department of Radiation Oncology, CHA Bundang Medical Center, CHA University, Seongnam, Korea

\section{References}

1. Jobsen JJ, ten Cate LN, Lybeert ML, van der Steen-Banasik EM, Scholten A, van der Palen J, et al. The number of metastatic sites for stage IIIA endometrial carcinoma, endometrioid cell type, is a strong negative prognostic factor. Gynecol Oncol. 2010;117:32-6

2. Kato T, Watari H, Endo D, Mitamura T, Odagiri T, Konno Y, et al. New revised FIGO 2008 staging system for endometrial cancer produces better discrimination in survival compared with the 1988 staging system. J Surg Oncol. 2012;106:938-41.

3. Connell PP, Rotmensch J, Waggoner S, Mundt AJ. The significance of adnexal involvement in endometrial carcinoma. Gynecol Oncol. 1999;74:74-9.

4. Denschlag D, Tan L, Patel S, Kerim-Dikeni A, Souhami L, Gilbert L. Stage III endometrial cancer: preoperative predictability, prognostic factors, and treatment outcome. Am J Obstet Gynecol. 2007;196:546.e1-7.

5. Lum MM, Belnap TW, Frandsen J, Brown AP, Sause WT, Soisson AP, et al. Survival analysis of cancer patients with FIGO stage IIIA endometrial cancer. Am J Clin Oncol. 2015;38: 283-8.

6. Havrilesky LJ, Secord AA, O'Malley DM, Broadwater G, BaeJump V, Cohn DE, et al. Multicenter analysis of recurrence and survival in stage IIIA endometrial cancer. Gynecol Oncol. 2009;114:279-83.

7. Lewin SN, Herzog TJ, Barrena Medel NI, Deutsch I, Burke WM, Sun X, et al. Comparative performance of the 2009 international Federation of gynecology and obstetrics' staging system for uterine corpus cancer. Obstet Gynecol. 2010;116: 1141-9.

8. Choi EC, Kim JH, Kim OB, Byun SJ, Park SG, Kwon SH. Postoperative radiotherapy for endometrial cancer. Radiat Oncol J. 2012;30:108-16.
9. Schorge JO, Molpus KL, Goodman A, Nikrui N, Fuller AF Jr. The effect of postsurgical therapy on stage III endometrial carcinoma. Gynecol Oncol. 1996;63:34-9.

10. Kuku S, Williams M, McCormack M. Adjuvant therapy in stage III endometrial cancer: treatment outcomes and survival: a single-institution retrospective study. Int J Gynecol Cancer. 2013;23:1056-64.

11. Nakayama K, Nagai Y, Ishikawa M, Aoki Y, Miyazaki K. Concomitant postoperative radiation and chemotherapy following surgery was associated with improved overall survival in patients with FIGO stages III and IV endometrial cancer. Int J Clin Oncol. 2010;15:440-6.

12. Hogberg T, Signorelli M, de Oliveira CF, Fossati R, Lissoni AA, Sorbe B, et al. Sequential adjuvant chemotherapy and radiotherapy in endometrial cancer: results from two randomised studies. Eur J Cancer. 2010;46:2422-31.

13. Alvarez Secord A, Havrilesky LJ, Bae-Jump V, Chin J, Calingaert $\mathrm{B}$, Bland $\mathrm{A}$, et al. The role of multi-modality adjuvant chemotherapy and radiation in women with advanced stage endometrial cancer. Gynecol Oncol. 2007;107:285-91.

14. Kuoppala T, Maenpaa J, Tomas E, Puistola U, Salmi T, Grenman S, et al. Surgically staged high-risk endometrial cancer: randomized study of adjuvant radiotherapy alone vs. sequential chemo-radiotherapy. Gynecol Oncol. 2008;110:190-5.

15. Mariani A, Webb MJ, Keeney GL, Aletti G, Podratz KC. Assessment of prognostic factors in stage IIIA endometrial cancer. Gynecol Oncol. 2002;86:38-44.

16. AlHilli MM, Mariani A, Bakkum-Gamez JN, Dowdy SC, Weaver AL, Peethambaram PP, et al. Risk-scoring models for individualized prediction of overall survival in low-grade and high-grade endometrial cancer. Gynecol Oncol. 2014;133: 485-93. 
17. Marchetti C, Pisano C, Mangili G, Lorusso D, Panici PB, Silvestro $G$, et al. Use of adjuvant therapy in patients with FIGO stage III endometrial carcinoma: a multicenter retrospective study. Oncology. 2011;81:104-12.

18. Jobsen JJ, Naudin Ten Cate L, Lybeert ML, Scholten A, van der Steen-Banasik EM, van der Palen J, et al. Outcome of endometrial cancer stage IIIA with adnexa or serosal involvement only. Obstet Gynecol Int. 2011;2011:962518.

19. Lan C, Huang X, Huang Y, Xi S, Huang H, Feng Y, et al. The outcome and efficacy of adjuvant chemotherapy alone in patients with stage IIIA endometrial carcinoma with solitary adnexal involvement: a retrospective single-institution study. Gynecol Oncol. 2014;135:446-50.

20. Ashman JB, Connell PP, Yamada D, Rotmensch J, Waggoner SE, Mundt AJ. Outcome of endometrial carcinoma patients with involvement of the uterine serosa. Gynecol Oncol. 2001;82:338-43. 